\title{
Preface: Special Topic on Metal-Organic Frameworks (MOFs)
}

\author{
Xian-He $\mathrm{Bu}^{1 *} \&$ Jing-Lin $\mathrm{Zuo}^{2 *}$ \\ ${ }^{1}$ College of Chemistry, Nankai University, Tianjin 300071, China \\ ${ }^{2}$ Department of Chemistry, Nanjing University, Nanjing 210093, China
}

Citation: Bu XH, Zuo JL. Preface: Special Topic on Metal-Organic Frameworks (MOFs). Sci China Chem, 2016, 59: 927-928, doi: $10.1007 / \mathrm{s} 11426-016-5603-2$

Metal-organic frameworks, MOFs, are coordination networks with organic ligands and voids. At the fundamental level, MOFs epitomize the beauty of chemical structures and the power of combining organic and inorganic chemistry, two disciplines that are often regarded as disparate. MOFs have recently emerged as an extensive class of crystalline materials with ultrahigh porosity and internal surface areas. These properties of MOFs, together with the extraordinary degree of variability for both the organic and inorganic components of their structures, bring great promise for a large, diverse array of applications. MOF research has been one of the most active areas in chemistry with an extraordinary pace since the 1990s. Our search of the literature, through the Web of Science, using the keywords "metal-organic frameworks" showed that there were 30412 publications from 1990 to May 2016. Analyses of these publications in Figure 1 show that gas sorption, catalysis and fluorescence are the top three subjects of MOF research, while studies of MOFs for battery application and electrocatalysis have been very active lately.

The concerns about the environment and energy supplies have led to the investigations of MOFs for gas adsorption such as hydrogen and methane storage, carbon dioxide capture, and the selective separation of light hydrocarbons. MOFs as fluorescent sensors could detect many types of hazardous substances such as metal ions, negative ions, and explosives. Porous MOFs, with their high efficiency and retrievability, are considered as promising candidates for

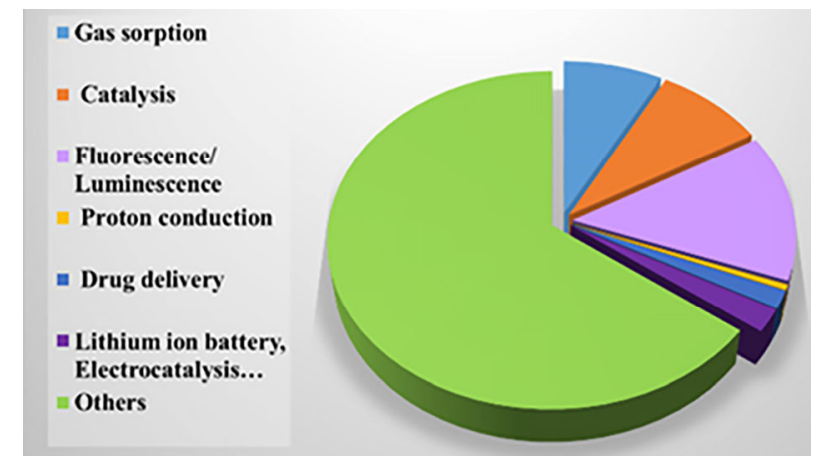

Figure 1 The ratio of the papers on the different MOF topics from 1990 to May 2016

catalytic reactions. Although these three topics are the most studied in MOFs, applications of MOFs in other areas, such as proton conduction, drug delivery, lithium ion and oxygen batteries, supercapacitors, electrocatalysis, and solar cells, have been very active.

This special topic of Science China Chemistry illustrates recent MOF research in several areas including magnetism, fluorescent sensors, and gas storage. Many scientists at the frontiers of MOF research have contributed reviews or research articles in this issue. Due to the limit of the space, it cannot cover all areas of MOFs. We hope that chemistry of MOFs and their applications will continue to attract many people from increasingly diverse disciplines to make contributions for a long time to come.

*Corresponding authors (email: buxh@nankai.edu.cn; zuoj1@nju.edu.cn) 


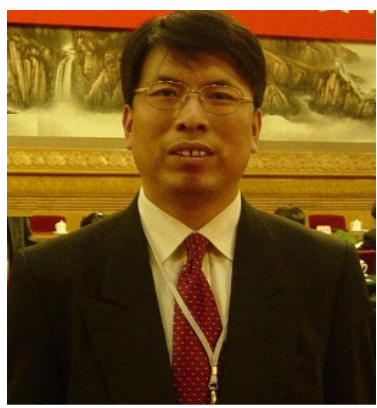

Prof. Xian-He Bu is a Cheung Kong Scholar Professor at Nankai University (from 2004). He obtained his B.S. and Ph.D. degrees from Nankai University in 1986 and 1992 under the supervision of Prof. Rong-Ti Chen. Since 1995, he has been a full professor at Nankai University. He is now serving as a director of Tianjin Key Lab of Metal and Molecule-Based Material Chemistry. His current research is focused on the syntheses and applications of multifunctional MOFs, crystal engineering, and magnetic materials.

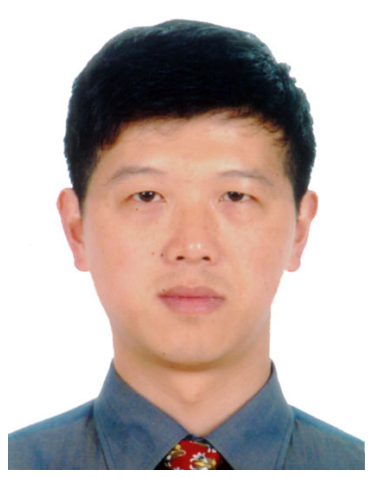

Prof. Jing-Lin Zuo received his B. S. (in 1990) and Ph. D. degrees (in 1997) from Department of Chemistry, Nanjing University. He was Research Assistant in the University of Hong Kong, and then postdoctoral research fellow (supported by Alexander von Humboldt fellowship) in Technical University Munich and Harvard University. Since 2004, he has been Professor in Nanjing University, China. His current research includes design, synthesis and properties of new information storage and electro-optical conversion material based on coordination complexes and organometallic compounds. 